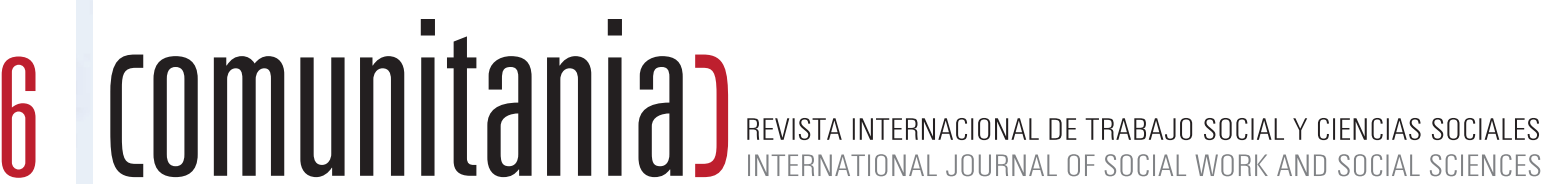

IGNASI BRUNET ICART | JOAN RODRÍGUEZ SOLER | BEATRIZ ESCUDERO BERZAL | PALOMA SERRANO POSTIGO LUIS MANUEL RODRÍGUEZ OTERO | MARTHA LETICIA CABELLO GARZA | MARÍA CONCEPCIÓN ARROYO RUEDA 


\title{
La obesidad paradójica: construcción de una imagen corporal contradictoria / Paradoxical obesity: building a contradictory
} body image

\author{
Martha Leticia Cabello Garza* y María Concepción Arropo Rueda** \\ * UANL. marthacabello@hotmail.com \\ ** UANL. aguaconflores@hotmail.com
}

\begin{abstract}
Abstrac:
This work resume the results of a phenomenological study on obesity about Mexican and Mexican-Americans participants (Cabello and Zúñiga 2007), selecting 18 candidates with average obesity and morbid obesity through an intentional criteria (9 women and 9 men Mexican and Mexican-Americans) who had a Body Mass Index (BMI) equal to or greater than 35, ages between 24 and 55 years. The central idea in the structure of the study was founded on lived experiences of participants; considering that each person creates, organizes and manage in a unique way their own experience. The contribution of the theoretical approach of Husserl's Phenomenological Perspective (1998) and Lazarus' emotions Theory (2000) were used as the lens through which was explained the lived experience of the research subjects. The results show that obesity generates paradoxes that allow participants to self-acceptance and "live happily in big bodies», which conduce into a positive self image; and the other hand, a perception that causes disputes conflict and frustration, especially whom start to gain weight after a stressful life event. The lived experience of these participants found out a positive connotation towards food that comes from eating patterns learned in the original family and a specific cultural context; and other negative connotation that comes of using food as a refuge in stressful life events, or dealing with unpleasant emotions. The results of the study are invited to a social intervention proposal for dealing with obesity focused on symbolic interaction theory; the key point is the assumption of the reflexive nature of people and their autonomy to take action through principle interactive determination. This proposal focuses on strengthening the skills of people with obesity through self-help groups, in order to generate new behaviors, attitudes, emotions and thoughts through a process of re-learning and redefinition of concepts that creates sustainable change in their life quality.
\end{abstract}

Keywords: obesity, phenomenology, Theory of emotions, Social Work.

\section{Resumen:}

Este trabajo retomó los resultados de un estudio fenomenológico sobre la obesidad entre mexicanos y México-americanos (Cabello y Zúñiga 2007). Se eligieron los discursos 
de 18 personas con obesidad media y extrema a través de un criterio intencional (9 mujeres y 9 hombres Mexicanos y México-americanos) que tuvieran un Índice de Masa Corporal (IMC) igual o mayor a 35, que su edad oscilara entre los 24 y los 55 años. La idea central en la estructura del estudio se basó en que aunque pueda existir cierto grado compartido en las experiencias de los participantes, que cada persona integra, significa y organiza de una manera única su experiencia. El aporte del enfoque teórico de la perspectiva Fenomenológica de Husserl (1998) y la teoría de las emociones de Lazarus (2000) fueron utilizadas como los lentes a través de los cuales se explica la experiencia vivida de los sujetos de investigación. Los resultados muestran que la obesidad genera paradojas que permiten a los participantes por un lado la autoaceptación "vivir felices en cuerpos grandes", que se traduce en una imagen positiva de sí mismo, y por la otra una percepción que les provoca conflicto y frustración, sobre todo aquellos que empiezan a ganar peso a partir de un evento estresante en la vida. La experiencia vivida por estos participantes encuentra una connotación positiva hacia la comida que viene de patrones alimenticios aprendidos en la familia de origen y en un contexto cultural determinado; y otra connotación negativa que se deriva del uso de la comida como refugio en eventos estresantes de la vida, o para afrontar emociones displacenteras. Los resultados del estudio invitan a una propuesta de intervención social para la atención de la obesidad basada en la Teoría del Interaccionismo simbólico, cuyo punto clave es la asunción de la naturaleza reflexiva de las personas y su autonomía para llevar a cabo acciones a través de principio de determinación interactiva. Esta propuesta está centrada en fortalecer las habilidades necesarias de personas con obesidad a través de grupos de ayuda mutua, con el fin de generar nuevas conductas, actitudes, emociones y pensamientos mediante un proceso de re-aprehendizaje y de resignificación de conceptos que generen cambios sustentables en su calidad de vida.

Palabras clave: obesidad, fenomenología, Teoría de las emociones, Trabajo Social.

\section{Article info:}

Received: 13/09/2012 / Received in revised form: 01/06/2013

Aceppted: 01/07/2013 / Published online: 15/07/2013

DOI: http://dx.doi.org/10.5944/comunitania.6.5/

\section{Introducción}

Uno de los más grandes retos a los que se enfrenta la sociedad mexicana en materia de salud es el sobrepeso y la obesidad de sus habitantes, este problema se ha agudizado a tal grado que se ha convertido en un grave riesgo para la viabilidad y estabilidad del sistema de salud en su conjunto. Su origen multifactorial y el factor de riesgo a desarrollar diversas enfermedades como la diabetes, enfermedades cardiovasculares o hipertensión arterial desde la infancia, así como los problemas psicológicos y sociales que se pueden presentar en las personas que padecen esta enfermedad, han convertido este problema de salud pública como prioritario en las 
agendas de salud (Bertrán 2009). Países en desarrollo, entre los que se encuentra México han triplicado las cifras de obesidad debido en parte a un aumento desmedido del consumo de comida rápida y económica de alto contenido energético, consecuencia de la globalización y del desarrollo tecnológico, y a una vida cada mes más sedentaria (OMS 2010). Ha sido tal la magnitud del problema en nuestro país, que México ocupa el segundo lugar en obesidad general y el primero en obesidad infantil (IMSS 2010; Calleja 2010). Según la última Encuesta Nacional de Salud en México (ENSANUT 2012), 22 millones de mexicanos presentan obesidad y casi una tercera parte de la población entre 5 y 11 años de edad presenta exceso de peso corporal, lo cual persiste como un gran reto a los sistemas de salud.

Aunque en la génesis de la obesidad se acierta gran evidencia científica que apunta a causas endógenas al individuo como el consumo excesivo de alimentos hipocalóricos y el sedentarismo, se encuentran muchos otros factores exógenos a las personas como aspectos económicos, políticos, culturales o psicológicos que directa o indirectamente, han influido en el problema de obesidad (OMS 2010; Shamah et al. 2007), creando un ambiente obesogénico con el que es necesario lidiar.

Los factores socioculturales que afectan la alimentación y la nutrición incluyen desde las tecnologías materiales, hasta las ideologías y símbolos implícitos. Así encontramos que en sociedades que presentan pautas nutricionales similares como son los mexicanos y los mexicoamericanos, los individuos tienden a adoptar los hábitos alimenticios que practican en el grupo social al que pertenecen; hábitos que se construyen y moldean dentro del contexto social, familiar, y económico en el cual se desenvuelve la persona, que son adquiridos desde etapas tempranas de la vida y responden no solo a la necesidad biológica de llenar el cuerpo de alimento, sino a las condiciones materiales y representaciones simbólicas que de ellas se pueda tener (Pérez, et al. 2007; Sedó 2005). Así, el estatus social que juega la comida y el simbolismo que rodea a la misma, le otorgan un énfasis particular, lo cual es en gran parte determinante de la obesidad. El presente trabajo muestra a través de los discursos de los participantes una connotación positiva hacia la comida como resultado de esas representaciones y creencias originadas en patrones alimenticios aprendidos en la familia de origen y en un contexto social determinado, y otra connotación negativa que se deriva del uso de la comida como refugio en eventos estresantes en la vida.

En las sociedades modernas, los cuerpos son reflejo de múltiples identidades. No obstante, pareciera que los cuerpos han llegado a ser más importantes que los sujetos pues es más frecuente escuchar describir a una persona por sus características físicas, que por su ser en sí mismo. Es decir, la imagen corporal es la primera descripción de los sujetos, antes de cualquier otro atributo (Gavilán 2005). En este sentido, las experiencias vividas de los participantes del estudio podrán ilustrar el impacto en la subjetividad a partir de la forma en cómo ellos mismos definen, configuran y reconfiguran su cuerpo y su identidad. 
Por otra parte, las distintas concepciones sobre el cuerpo que cada persona integra a partir de sus referentes socioculturales, se expresan a partir de la subjetividad, adquiriendo un carácter relacional e institucional. Pero además una dimensión social, que se encuentra en las representaciones sociales, los mitos, las creencias, y está atravesada por los discursos predominantes en el imaginario social (Ramírez y Anzaldúa 2005). La subjetividad "expresa la síntesis a nivel simbólico y de sentido subjetivo, del conjunto de aspectos objetivos, macro y micro, que se articulan en el funcionamiento social" (González 2007: 17). Dichos planteamientos toman relevancia en nuestro trabajo, dado que la imagen corporal se construye a partir de elementos individuales-subjetivos y dimensiones sociales, en donde se identifican discursos contradictorios, paradójicos que dan cuenta de las diferentes formas de subjetividad y también de distintas maneras de afrontar un fenómeno social como la obesidad. Desde hace tiempo se han venido realizando investigaciones sobre la obesidad y se han hecho descubrimientos con importantes implicaciones. En décadas recientes este interés ha sido impulsado por descubrimientos que varían de las causas genéticas a las económicas, sin embargo sólo una pequeña cantidad de publicaciones se han enfocado en la investigación cualitativa sobre la obesidad (Núñez 2007). Aún menor ha sido la investigación encaminada hacia la percepción que las personas obesas, tienen de las ideas, sentimientos y simbolismos relacionados con la comida.

Varios estudios muestran como las personas obesas utilizan la comida como refugio para compensar sus frustraciones, tristezas, necesidades y temores (Cabello 2010). Otros han hecho alusión a los efectos de la burla acerca del cuerpo obeso y cuentan de la insatisfacción por la imagen corporal y algunos desórdenes alimenticios (Fabian yThompson 1989; Lunner et al. 2000). Sin embargo, la influencia que los otros ejercen sobre la visión de los cuerpos y los atributos físicos, también puede influir en forma positiva. Estudios relacionados con la imagen del cuerpo han encontrado que la pérdida de peso puede no estar asociada con sentimientos negativos hacia su cuerpo, como Thompson y Heinberg (1999) quienes afirman que la mayor satisfacción en la imagen del cuerpo puede ocurrir en ausencia de la pérdida de peso.

Estudios como el realizado por Cabello y Zúñiga (2007), encontraron que hay quienes indiferentes al sobrepeso, afirman tener una imagen positiva de su cuerpo y vivir felizmente en cuerpos grandes; perder peso no les preocupa, están contentos y cómodos con el tamaño de sus cuerpos y con su estilo de vida, afirmando además el tener relaciones sociales positivas. Otras investigaciones afirman que existen efectos positivos a partir de la obesidad que se producen sobre todo cuando las madres y amigas de adolescentes y jóvenes han transmitido mensajes positivos acerca del cuerpo con sobrepeso (Pelican et al. 2005).

Se ha documentado también que valores familiares, tales como vínculos de relación adecuados y expectativas positivas de los padres, se constituyen en "factores de protección" para las personas obesas y se establecen a la vez como un soporte 
emocional fundamental para hacerle frente a los discursos negativos de la obesidad que generalmente predominan en el contexto social. El enfoque fenomenológico de este estudio proporcionó una manera de estudiar las perspectivas individuales relacionadas con la experiencia altamente personal de ser una persona obesa. El propósito final fue entender el contexto particular en el cual estos eventos ocurren y anticipar qué pensamientos, ideas o sentimientos se manifiestan en los participantes en relación con el estatus de su peso.

\section{Teoría de las emociones de Lazarus}

El ser humano invariablemente desarrolla estrategias de afrontamiento ante situaciones adversas y que, en el caso de la obesidad, la aceptación y bienestar puede ser producto de un patrón de afrontamiento que busca cambiar una situación conflictiva. En este sentido, Lazarus (2000) plantea que la función más importante del afrontamiento es regular las emociones generadas a partir de una situación conflictiva. Si bien en algunas personas la obesidad produce una transgresión a la identidad, a la autoestima y a la auto-confianza de la persona, adoptar una actitud positiva frente a ello, regula la tensión y las emociones relacionadas con ella. En otras palabras, cuando la integridad se ve amenazada, las personas luchan para evitar que ocurra el daño o para poder vencerlo (Lazarus 2000: 197).

Desde el planteamiento de Lazarus (2000), se puede interpretar que cuando las personas obesas sienten que poco o nada pueden hacer para bajar de peso, cuando sus múltiples intentos para lograrlo han fallado, las estrategias de afrontamiento centradas en la emoción se construyen para controlar la angustia y pasar de una experiencia amenazadora a otra más serena o positiva.

Este tipo de afrontamiento centrado en la emoción tiene dos vertientes: a) la evitación y, b) la revaluación del significado personal. Según Lazarus (2000): en la evitación las personas intentan no pensar en aquello que les preocupa, adoptando toda clase de estrategias que puedan distraerlas. Paradójicamente, las personas que presentan algún problema pueden ocupar tiempo preparando complicadas comidas, o haciendo ejercicio, durmiendo más de lo habitual o durmiendo mal. Pero, lo que hace que la evitación sea una inadecuada estrategia de afrontamiento, es que aunque las preocupaciones pueden parecer menos graves durante un tiempo, los problemas no desaparecen hasta que se enfrentan o se resuelven. La otra forma de afrontamiento que es más saludable, sería cambiar el significado personal de lo ocurrido, evaluándolo de manera menos amenazadora; es decir, una estrategia de afrontamiento centrada en la emoción que a la larga se puede convertir en la estrategia más eficaz (Lazarus 2000).

Tomar en cuenta la perspectiva de las emociones resulta importante en el caso de este estudio, pues consideramos que las actitudes y respuestas que damos ante la 
mayoría de los eventos de la vida cotidiana se centran en las emociones. Además, creemos que las respuestas emocionales se producen a partir de un componente biológico, pero también social, que determina el tipo de emoción y el tipo de acción que se desencadena.

En el caso de la obesidad, las personas se asumen de una manera distinta, dependiendo de cómo se construyan como personas y de cómo configuran su imagen corporal. Pero tal construcción está influida fuertemente por la información y el conocimiento tanto científico, como de sentido común que circula en el contexto a que pertenecen dichas personas. Es decir, su self, siempre va a estar definido en función del Otro. De igual forma, las estrategias de afrontamiento que utilizan los participantes de este estudio, también están centradas en la emoción, y esa reacción emocional, adicionalmente surge en complementación de los discursos que la originan.

Siguiendo con este enfoque teórico, el principio de cambiar el significado personal para reducir la aflicción por una determinada problemática, se aplica a los estados emocionales como ansiedad, culpa, vergüenza y depresión, que son emociones presentes en la imagen negativa de la obesidad. No obstante existen otras emociones hacia su cuerpo de autoaceptación, siendo personas alegres, positivas que gozan y disfrutan de la comida; así, las ideas, emociones y comportamientos que surgen alrededor de la obesidad, pueden ser ambivalentes y paradójicas de acuerdo a las construcciones sociales que de manera individual y social se presenten en una cultura determinada.

\section{Abordaje cualitativo-fenomenológico del estudio}

Este trabajo se abordó desde una perspectiva fenomenológica, que fue utilizada para explorar la experiencia vivida de personas con obesidad. En términos simples, la fenomenología es el estudio interpretativo de la experiencia humana. El objetivo es estudiar y aclarar las situaciones humanas, eventos, significados y experiencias "que ocurren espontáneamente en el curso de la vida cotidiana" (Von Eckartsberg 1998: 204). El objetivo es "una descripción rigurosa de la vida humana tal como es vivida y reflexionada" (Pollio et al. 1997: 156).

El padre fundador de la fenomenología, el filósofo Edmund Husserl, creía que bajo el flujo cambiante de la experiencia humana, había ciertas estructuras invariables de la conciencia, las cuales podrían ser identificadas a partir del método fenomenológico. Por su parte Polkinghorne, (2004), señala que el mundo de la vida se refiere al mundo cotidiano como es vivido por todos nosotros, a priori de las explicaciones e interpretaciones teóricas de cualquier tipo. El mundo de la vida comprende emociones, motivaciones, símbolos y significados, empatía y otros aspectos subjetivos relacionados con la evolución natural de la vida del individuo. Este es el punto de partida de la reflexión fenomenológica. Husserl (1965) deseaba desarrollar una cien- 
cia del fenómeno que pudiera clarificar como los objetos aparecen en la experiencia y se presentan a la conciencia de las personas.

De acuerdo a Husserl (1998), las personas conocen el mundo a través de dos aspectos inseparables: a) la captación intuitiva, donde la persona entra en contacto con el mundo por medio de sus sentidos, representando un conocimiento directo aunque incompleto, ya que la persona no puede estar exenta de tener cierta perspectiva en su percepción, y b) la integración significativa, donde a través de la reflexión, la persona capta los fenómenos no como se presentan a sus sentidos, sino como se presentan a su conciencia. Estas son las premisas que guiaron el presente estudio. Estos dos aspectos del conocimiento del mundo, expresan que los objetos que la persona percibe (incluyendo aquí la percepción de otras personas e inclusive, la percepción de sí mismo), existen, en la forma específica en que aparecen a través del significado que la misma persona les brinda.

Según González (2003), la fenomenología es una filosofía que se ocupa de la conciencia, de los procesos mentales mediante los cuales conocemos las cosas y sabemos que las conocemos. Además permite formar un conocimiento específico, natural, de sentido común y práctico de las experiencias de cada uno de los participantes del estudio, experiencias que se constituyen a partir de las vivencias, conocimientos, modelos de pensamiento e información, que se recibe y transmite por la tradición, la educación y la comunicación social (Martínez 2006).

El abordaje fenomenológico favoreció el entendimiento del problema desde las percepciones del propio actor, describiendo cuáles son los motivos, las creencias, la conducta, lo que dicen, hacen y qué es lo que existe detrás de las acciones que se encuentran en el proceso de bajar y mantener el peso. Esta perspectiva tiene como propósito primordial centrarse en la experiencia personal y es flexible en su estructura para comprobar la multiplicidad de las experiencias cotidianas (Álvarez-Gayou 2003; Husserl 1998) “La fenomenología considera que los seres humanos están vinculados con su mundo y pone énfasis en su experiencia vivida, la cual aparece en el contexto de las relaciones con objetos, personas, sucesos, y situaciones" (Álvarez-Gayou 2003: 86).

La fenomenología comprende al ser humano en su mundo, a partir de sus opiniones, ideas y sentimientos, trata de descubrir cómo es que el otro ve, siente, piensa sobre los acontecimientos vividos. Esta perspectiva, es para Husserl (1998), vida trascendental o esencial; es un modo de vivir la misma vida, sin salirse de ella hacia una causa trascendente, pero de modo radicalmente distinto, evidenciando la esencia de las cosas. Desde un punto de vista fenomenológico las personas muestran actitudes naturales de acuerdo a la forma en que presuponen el mundo, considerando en este caso sus emociones, como reales e incuestionables. Desde este enfoque resultó interesante conocer las experiencias vividas de ser una persona con obesidad y lograr un mejor entendimiento de los sentimientos y las emociones paradójicas que viven los participantes del estudio. 


\section{Metodología}

El presente estudio cualitativo utilizó un enfoque fenomenológico. Este abordaje posibilitó la obtención de una imagen realista y fiel de las personas entrevistadas, identificando pautas de pensamiento y abordando un número limitado de casos bajo una perspectiva integral permitiendo el acceso a sus esferas privadas (Denzin y Lincoln 2003; Husserl 1999).

El enfoque fenomenológico aplicado en este estudio tuvo la particularidad de obtener los datos directamente de los puntos de vista de las experiencias vividas por los sujetos que presentan obesidad, así como comprender los significados e interpretaciones de su experiencia en bajar de peso y es ahí que se pretende descubrir cuáles son los fenómenos ocultos y en particular los significados no manifiestos, para así tratar de examinarlos y relatarlos (Martínez 2006; Rodríguez et al. 1999).

Este trabajo retomó los resultados de un estudio fenomenológico sobre la obesidad entre Mexicanos y México-americanos (Cabello y Zúñiga 2007), eligiendo los discursos de 18 personas con obesidad media y extrema a través de un criterio intencional ( 9 mujeres y 9 hombres Mexicanos y México-americanos) que tuvieran un Índice de Masa Corporal (IMC) igual o mayor a 35, que su edad oscilara entre los 24 y los 55 años. Las razones por las que se eligió entrevistar a mexicoamericanos entre todas las minorías raciales y étnicas en los Estados Unidos no fue sólo porque tienen la más alta frecuencia de individuos con sobrepeso en este país, sino porque comparten antecedentes culturales y hábitos alimenticios similares con los mexicanos. En el caso de los mexicanos, deberían vivir en México y los México-americanos deberían haber nacido en los Estados Unidos o vivido ahí desde la infancia. Se excluyeron aquellos participantes con problemas metabólicos, hormonales o que estuvieran ingiriendo medicamentos con efectos en el peso.

La información fue recolectada a través de entrevistas cualitativas semiestructuradas, en español. En los Estados Unidos, se obtuvo la cooperación del Centro para Estudios México-americanos (Center for Mexican American Studies) y de algunas iglesias católicas para el contacto con los entrevistados; y en México, se contactó con algunos centros de autoayuda y de la población en general. La técnica de bola de nieve, así como la colaboración del público, fueron utilizadas para identificar a los participantes. En México, las entrevistas se realizaron en los municipios de Monterrey y San Nicolás de los Garza, en el Estado de Nuevo León; y en la ciudad de Piedras Negras, en el Estado de Coahuila; en los Estados Unidos, las entrevistas se realizaron en Arlington, Dallas, Eagle Pass, Laredo, McAllen, Mesquite, Mission y Weslaco, en el Estado de Texas. El punto de saturación teórica fue el criterio utilizado para determinar el número de participantes procurando que fuera un número comparable de participantes de acuerdo al sexo. 
El protocolo para este estudio fue revisado y aprobado por el IRB (Institutional Review Board) de la Universidad de Texas en Arlington. Se firmó un consentimiento informado para cada uno de los participantes, donde se aseguró el anonimato, y asignando seudónimos en cada caso. Se realizaron transcripciones textuales de las grabaciones de las entrevistas. Una herramienta muy útil, utilizada para el manejo de los datos cualitativos, fue el AskSam que es un sistema de administración de base de datos flexible, procesador y buscador de textos. Para demostrar la validez en la recolección cabal y auténtica de los datos, se hizo una precisión factual de la narración de los participantes, intentando explicar los significados alternativos. Estrategias tales como triangulación de datos, análisis negativo de casos y revisión de miembros fueron utilizados para incrementar el rigor del estudio (Maxwell 2009).

\section{Resultados. La evidencia empírica}

Los resultados obtenidos muestran un entendimiento comprehensivo, coherente con las perspectivas teóricas elegidas. Para obtener mayor claridad, se presentan en categorías empíricas que dan cuenta de las experiencias vividas por los participantes, en las cuales están plasmadas las ideas, las creencias y las emociones paradójicas que genera el fenómeno de la obesidad.

\section{Discursos relativos a emociones negativas de su imagen corporal}

\section{- Tristeza y Depresión}

La tristeza muchas veces se confunde con la depresión, pero es la tristeza la que predomina cuando se sufre una pérdida importante. El significado personal de la tristeza por la pérdida, puede variar de persona a persona. El tipo de pérdidas van desde la pérdida de la salud, la pérdida de los seres queridos, o bien la pérdida de un estatus o posición social que otorga identidad (Lazarus 2000). En el caso de las personas obesas, la pérdida se relaciona con la carencia de una imagen estética, de belleza, donde el no tener una figura que resulte atractiva, genera tristeza y aislamiento.

(...) o sea hay períodos en que de repente te ves como eres y causa un shock emocional muy fuerte.....entonces estuve tomando terapia para poder asimilar, vaya, es decir cada vez que a mí me pasaba, o sea que yo me daba cuenta realmente de cómo era, a mí me daban depresiones de no salir de mi cuarto o sea de que me daba miedo salir, porque me veía horrible, o sea por decir había andado todo el día afuera pero ya en la noche que me daba cuenta de eso, al otro día yo no quería salir y cosas así...(Roberto, 287 kilos).

Entendemos, en este relato, cómo la entrevistada construye a partir de su imagen corporal, sus emociones hacia sí misma y a la vez en la relación con los otros. En su 
lógica, la imagen que observa de sí mismo, genera rechazo y vergüenza. Pero como se configura ésto? es sólo a partir de su percepción? o es también a partir de los discursos que escucha? y, de dónde provienen tales discursos? Veamos en el siguiente fragmento

Creo que si regresamos a 1989, perdí mi trabajo y dejé la universidad, sin embargo, mucha gente dice que fue más bien por una depresión que tuve cuando terminé con una chica... [en tono muy triste afirma] ella se metió de monja... Creo que eso fue un detonador, después me volví una persona más introvertida, mas inactiva, solitario, desde entonces yo he aumentado casi 68 kilos. (Roberto, 287 kilos).

La percepción de rechazo está presente en distintos entrevistados; para ellos es difícil comprender que este se instala en una información sobre la obesidad que circula en su mundo social, en el mundo de la vida. Escuchar en los medios masivos de comunicación, en la escuela, en el trabajo, con los amigos, que la gente atractiva y deseable es la gente delgada, tiene un peso importante en las formas de concebir sus cuerpos.

\section{- Autoagresión}

En algunos casos, cuando surge la depresión, se genera no una emoción concreta, sino más bien una combinación de varias emociones como el enojo, ansiedad o frustración. Lo más complicado, en el caso del enojo es cuando resulta contra sí mismo; las personas con sobrepeso, en ocasiones manifiestan su enojo, agrediéndose a sí mismas, como lo ilustra el siguiente discurso:

(...) finalmente como cuando tenía más o menos esa edad me harté de que todo mundo me estuviera diciendo que baje de peso y que todo mundo tuviera el derecho digamos de estarme agrediendo, en ese sentido entonces opté por agredirme yo misma, y me agredí más de lo que cualquier persona pudiera agredirme, esa era una especie de protección, entre esas cosas, fui adicta a las anfetaminas.... (Marcela, 122 kilos).

El tema de la obesidad es un tema cuya significatividad es social (Shutz 1993), en este caso, Marcela, como muchas personas que no encuentran una forma más sana de enfrentar un problema de falta de aceptación, es asignada por ciertos estándares de cómo debe ser el cuerpo femenino. Entendemos entonces, que el problema referido no proviene del sujeto en particular que tiene un cuerpo obeso, sino de ciertas estructuras sociales que le son impuestas.

Por otra parte, las situaciones estresantes o de angustia generados por ciertos sucesos de vida, también influyen para que algunas personas se refugien en la comida. Los eventos estresantes en la vida de los entrevistados aparecen como un elemento importante en el desarrollo de la obesidad, quienes manifiestan habilidades inadecuadas para manejar el estrés psicológico (Cabello y Zuñiga 2007). 


\section{- Compulsión}

En un evento estresante donde se come de manera compulsiva, las personas ingieren grandes cantidades de comida mientras que sienten que no pueden controlar cuánto están comiendo (Caldwell y Kimball 2001). En este sentido, Ball y Lee (2000) establecieron la hipótesis que el estrés ejerce un rol significativo en la etiología de los desórdenes alimenticios. Estudios relacionados con este problema, apoyan el hecho que algunos individuos comen en respuesta al estrés emocional, es decir comen compulsivamente, y por lo tanto tienen un mayor riesgo de ser obesos (Decaluwé et al. 2003; McFarland y Backer-Bauman 1988).

En las narraciones de los participantes, se encontró que eventos relacionados con alguna pérdida importante, o cambios significativos en las circunstancias de su vida tales como un divorcio, pérdida de una relación o enfermedad, fueron algunos de los eventos traumáticos que precipitaron el comportamiento de comer compulsivamente, como lo muestra el siguiente relato:

Yo lloraba y comía..., fijate que me acuerdo muy bien,.... y mis amigas se ríen ahora, ....pero cuando estaba pasando por lo del divorcio, estaba yo en una escuela donde éramos puras mujeres y entonces estábamos a la hora del recreo y yo "llore" y "llore" y todas lloraban también, pero yo.... comiendo... de repente cuando estábamos en el torbellino, no me hicieron el comentario, pero como a los seis meses me decían: tú estabas llorando y a todas nos impactaba que nosotras llorábamos y no comíamos, pero tú estabas llorando y no dejabas de comer,... ¿Puedes creerlo? (Leslie, 103 kilos).

Muchas de las personas inician el consumo excesivo por la comida, como respuesta a una pena o un cambio que no pueden aceptar. Según Nakken (1999), la adicción a la comida ofrece a estas personas otra manera de enfrentarse con el problema. Según Cabello (2010), en su estudio todos los entrevistados que iniciaron su aumento de peso por un evento estresante en la vida, literalmente expresaron que se refugiaron en la comida después del suceso. El caso de Caly, confirma lo anterior al narrar cómo inicia en mayor medida su aumento de peso al tener un evento estresante, que en este caso fue la muerte de un familiar, y donde su única salida en ese entonces era la comida.

"Mire hace 12 años me atropellaron a mi hijo y no pos subí más de peso.... si subí más y más y más, porque cuando murió pesaba como 104...lo que si es que yo me he fijado que entre más tengo problemas más como y como y como.. Y yo siento que aquello me hace daño y sigo comiendo o sea que ya no es hambre...claro que pos me queda remordimiento porque yo sola pienso..." (Caly, 130 kilos).

Generalmente, las personas que sufren una pérdida tardan un cierto tiempo en asimilarlo, en aceptar y adaptarse a su nueva situación. Según Lazarus (2000), la diferencia entre resignación y aceptación es sutil. La persona obesa comiendo de más, contrarresta el nivel de estrés que siente profundamente, las necesidades emo- 
cionales frecuentemente producen un deseo urgente y compulsivo de ingerir alimentos. Leslie demostró este tema cuando recapituló un pasaje de su vida:

Soy de las personas que le doy frente a los problemas comiendo, por ejemplo yo sufrí mucho cuando el trasplante de mi hermano que entró en estado de coma pero yo recuerdo que yo comía donde sea... hasta en el hospital... yo no soy como algunas personas que no comen cuando tienen un problema, pero yo sí... (Leslie, 103 kilos).

El caso de Leslie permite reflexionar que es muy difícil que de manera consciente ella decidiera comer al tiempo en que recrea su experiencia ante los demás. Pareciera que con la comida, la entrevistada quisiera recuperar el afecto perdido, pues como bien sabemos, la comida desde un entendimiento psicoanalítico representa la satisfacción de una necesidad primaria: el afecto.

\section{- Devaluación y deterioro}

Las personas obesas pueden asimilar, aceptar o resignarse ante la situación vivida. Muchos de ellos aceptan o se resignan a su imagen corporal; sin embargo, la confianza en sí mismos afecta su identidad y su autoconcepto. Desde un punto de vista fenomenológico, el sentido de autoestima es un importante constructo en la identidad de una persona, además de ser una poderosa influencia en la conducta y en el estado emocional (Pelican et al. 2005). La influencia que las personas significativas tienen sobre la visión del cuerpo adquiere importancia en la autovaloración de las personas.

En relación a lo anterior, Mruk (1999) plantea que la autoestima tiene dos aspectos interrelacionados: un sentido de eficacia personal y un sentido de valor personal; ello integra la auto-confianza y el auto-respeto; es decir, se cuenta con la convicción de que una persona es competente y valiosa en la vida. Algunas personas obesas, al fracasar en su intento por disminuir su peso, pierden autoestima, y el estigma por la obesidad cobra importancia, mostrando ante los demás y ante sí mismo un "self" devaluado y deteriorado.

(....) exacto, siento que nunca me he aceptado gordo y he aprendido a vivir como obeso, pero nunca me he aceptado y eso pues me ha acarreado a lo mejor a veces problemas interpersonales con la gente con quien he convivido, o sea no soy muy afecto a las bromas de gordos o que tú mismo te hagas bromas de gordos... yo les decía pos si no es un circo, que la gente no te vea como que es un circo, que tienes un problema, un enfermedad, digo pos últimamente se le ha llamado enfermedad...(Roberto, 287 kilos).

(...) porque yo me cohíbo, no puedo hablar con las personas bien, y más si me gusta una persona, un muchacho. Yo puedo vacilar y hablar y jugar con personas que no me encuentro atraída, pero si me gusta un muchacho siento que no puedo hacerlo... yo siento que no que no merezco. (Laura, 105 kilos). 
Algunas de las personas afectadas por su sobrepeso, no son bien consideradas en la sociedad, y muchas de ellas tienen una pobre imagen de sí mismos y expresan sensaciones de inferioridad y rechazo. Los sentimientos de inferioridad y poco valor por sí mismo, generan también otras emociones frecuentes en el fenómeno de la obesidad: la culpa y la vergüenza.

\section{- Culpa y Vergüenza}

Desde la perspectiva construccionista, la emoción de la culpa se relaciona con sentir que se ha transgredido un código moral que forma parte de los valores de las personas; las personas que experimentan culpa, no necesariamente incurren a algo moralmente malo, sino que creen que lo han hecho. La emoción de la culpa se centra en el sentimiento subjetivo de culpabilidad (Lazarus 2000). En el caso de las personas con sobrepeso, la culpa sobreviene porque se sienten incapaces de trascender su obesidad, percibiendo su imagen corporal como algo que los devalúa ante los demás, por lo que se sienten discriminados o rechazados. Como lo narra Marcela (122 kilos) "(...) yo sé que tengo muchas marcas y muchos traumas que yo sé que no me voy a poder quitar, sin embargo yo he estado trabajando en las que me joden más tenerlas y una de ellas es la culpa..."

Según Lazarus (2000), muchas veces puede haber confusión entre la culpa y la vergüenza; el argumento central de la vergüenza, es no estar a la altura del ideal personal o del ego. Vivir en desacuerdo con el ideal personal, es una manera de evitar la vergüenza; parece que el objetivo subyacente que funciona en la vergüenza es evitar la crítica, el rechazo o el abandono social. En este tenor, es interesante ver cómo las personas que luchan contra la vergüenza prefieren "esconderse del mundo", ya sea mostrando una buena fachada, o bien evitando los contactos sociales. En las personas con sobrepeso, es común ese sentimiento de vergüenza, dado que el ideal de todo ser humano es tener un cuerpo estético y atractivo, pero que sobre todo cumpla con los estándares sociales creados por la cultura. Los ejemplos siguientes dan cuenta de cómo surge esta emoción en algunos participantes:

Pues mira yo como y luego estoy muy apenada, muy mortificada por mi falta de voluntad y...o sea como algo y luego me estoy lamentando, ni lo estoy saboreando, pero yo pienso que me falta que esté alguien conmigo llevando la dieta... y si la hacemos, como ahora que la hace mi vecina conmigo... (Roxana, 130 kilos).

Era muy asqueroso, pero bueno, y me daba de lonche una manzana o una naranja, lo cual a mí me daba demasiada vergüenza porque era como decirle a todo el mundo que estaba a dieta y que estaba gorda entonces hacía todo lo posible por no comérmelo ( Marcela, 122 kilos).

De nuevo surge la preocupación por la aceptación en este relato. Nos vemos reflejados en el Otro, nos construimos en base a la mirada del Otro. Y si esos "otros" rechazan la obesidad porque ésta no es el "ideal" de cuerpo que se promueve en 
nuestra cultura, entonces desarrollan estrategias para evitar la vergüenza de no cumplir con la expectativa creada acerca de ello.

(...) Mmmm... Bueno, como persona si soy alegre nomas que con el sobrepeso si me siento amargadita, o sea porque a veces quisiera ponerme alguna ropa y cambiar mi carácter... antes era más alegre ahora me he hecho más calmada, no me gusta salir mucho ...no me gusta ir a los bailes, antes me fascinaba ir a los bailes (Elena, 232 kilos).

La tendencia a "esconderse" de esta participante y de rehuir las relaciones sociales, se enmarca en el contexto de la vergüenza, de no querer enfrentar comentarios desagradables que la ponen de frente al problema de la obesidad. Entonces, al no sentirse capaz de afrontarlo, es mejor esconderse, es mejor aceptar que se es una persona inadecuada para la convivencia social y quedarse en casa.

Muchos de ellos reaccionan como la sociedad espera de ellos, es decir, se sienten mal o inadecuados, a veces generan sentimientos de baja autoestima o inseguridad ante la convivencia social, tal como se mostró en los discursos anteriores. Surge entonces una "normalización" de la imagen socialmente devaluada de la obesidad. Sin embargo, de manera paradójica, se encuentran personas que, después de una larga experiencia de sufrimiento y rechazo por los demás, adoptan una posición diferente ante el problema, reconociendo el esfuerzo que realizan para bajar de peso y cuestionando las formas en que se ha abordado la cuestión de la obesidad.

\section{- Enojo, resistencia y agresividad}

Algunas participantes especialmente mujeres, manifiestan emociones de enojo, rebelándose ante un imaginario social que las coloca como personas sin valor. En muchas ocasiones, las personas obesas ante los discursos que estigmatizan y devalúan sus cuerpos, no se ajustan a los estándares socialmente "adecuados", adoptan diferentes actitudes como el rebelarse, mostrarse indiferentes, enojados o combativos ante situaciones conflictivas surgidas en torno a su figura obesa.

(...) no te imaginas la cantidad de veces que se me rodaron las lagrimas de estar oyendo que tenías que estar a dieta toda la vida, que tenías que cuidarte toda la vida, etcétera y todo se veía tan eterno y es que tú no sabes la cantidad de veces que yo lloré ante un chocolate...y yo me preguntaba: ¿por qué "X\#\#\&" yo no me puedo comer un chocolate?.. Y es que tú crees que porque estas dada de alta ya puedes comer de todo! (Leslie, 103 kilos).

El placer que otorga cierto tipo de comida, se contrapone con los efectos que ésta puede tener en el peso de las personas. La persona con obesidad, aunque sabe inconscientemente que comer es una actividad satisfactoria, que le genera placer o le recuerda la protección de sus padres; cuando se somete al juicio social, a las críticas y a la impopularidad, entra en un estado emocional contradictorio que puede ir del enojo a la ansiedad. Esta ambivalencia es muchas veces el disparador que promueve el que las personas obesas busquen ayuda terapéutica. 
(...) como que mis relaciones cambiaron, o sea yo era como que....yo sentí que yo era muchas cosas y que esas cosas no valían absolutamente nada, pero cuando yo no tengo sobrepeso todas esas cosas valen, entonces hay una sobre valoración de mi misma, y hay un desprecio por los demás porque llega un punto en el que dicen jtu eres súper inteligente y tu eres lo otro i....ah..... Y para que tú te dieras cuenta que yo soy súper inteligente tuve que bajar de peso...pues sabes qué?...vete a \#\$\%\#\%\&!... si tu no fuiste capaz de valorarme cuando yo tenía sobrepeso, o sea... ahorita tú no me interesas... (Marcela, 122 kilos).

En los textos siguientes y retomando el concepto de resistencia de Focault (1979), da cuenta de cómo se puede llegar a de-construir el discurso hegemónico de la obesidad e intentar lograr una nueva identidad a partir de una actitud de enojo, pero también una actitud reflexiva y crítica que hace posible discursos diferentes en la misma participante.

(...) Bueno la concepción propia o contigo mismo es una construcción cultural, entonces yo pienso que en el momento en que tu para sentirte bien contigo misma necesitas autodestruirte, entonces estamos hablando de una falla social importante, comerciales que saca el seguro [se refiere al Instituto Mexicano del Seguro Social, una institución oficial en México para atención de la salud ] sobre el sobrepeso, son por ejemplo en este sentido... la sociedad está muy en la idea o sea todo este rollo que se desarrolla en los 80's con la estúpida de Jane Fonda y todo ese rollo de que si estas gordo es porque no te quieres, o porque no tienes fuerza de voluntad, el que está gordo es porque quiere tener sobrepeso o sea este tipo de cosas afecta en el sentido que te ven porque estas inmersa en una sociedad en que si a mí me gustas o no me gustas equis, si tienes esta imagen vendida entonces resulta que quien tiene sobrepeso no nada más tienes sobrepeso sino que además eres feo, y además de ser feo eres estúpido porque estas así porque quieres, no te quieres a ti mismo .....o sea una serie de adjetivos innecesarios y no reales que se le han ido adjudicando a la persona obesa, entonces todo esto así como se le vende al público en general, se la vende a la persona obesa, si no me voy a sentir bien conmigo misma y como tengo sobrepeso no valgo ni madre (Marcela 122 kilos).

Los cambios que surgieron en la forma de explicarse a sí misma el problema del sobrepeso en esta participante, se relacionan con el haber tomado parte en procesos terapéuticos, grupos de autoayuda, documentarse sobre el problema, etc.; es decir, ella exploró diversos "entendimientos" acerca de la obesidad, que le permitió construir nuevas explicaciones. Sin embargo el siguiente discurso de la misma entrevistada es un ejemplo del sentimiento de culpa provocado por su obesidad y que nos refleja la intención de modificar esa percepción:

(...)... la culpa te friega y te "desmadra", y esa ha sido sobre lo cual es una de las cosas por las que he estado trabajando ...o sea yo no me puedo sentir culpable por estar comiendo, o sea que si me discriminan en algún aspecto yo no debo sentirme culpable por eso, no puedo sentirme culpable...( Marcela 122 kilos)

En sus palabras vemos cómo la participante va tomando conciencia del daño que le han hecho las opiniones sobre la obesidad, y cómo al cuestionarlos se desprende de una posición en la que se ha visto sometida y sujeta a los discursos discriminatorios de 
la sociedad. El espacio terapéutico donde ella ha aprendido a cuestionar las supuestas "verdades" sobre la obesidad, han creado otras "verdades" más convincentes y gratificantes que están dando pie a una nueva identidad, a un sí mismo diferente. Como plantea Gergen (2006), nuestro sí mismo se conforma en un espacio intersubjetivo, interrelacional, a partir de las conversaciones y narrativas que construimos con los otros de nosotros mismos.

\section{Discursos ligados a emociones positivas de su imagen corporal}

No se necesita estar delgado para ser feliz; parece que la felicidad no depende del peso, sino de muchas otras circunstancias que nos rodean. Hay personas que son felices por sí mismas y otras necesitan estar rodeados de otras circunstancias para alcanzar la felicidad. Una persona con problemas de obesidad puede ser tan feliz o infeliz como una persona de peso normal (Cabello 2010). Un hallazgo fundamental de esta investigación fue que en los discursos afirman textualmente verse bien frente al espejo y decirse a sí mismos que se ven y se sienten bien con sus cuerpos. Para varios entrevistados la felicidad no depende del peso, sino de muchas otras circunstancias que los rodean. Hay personas que son felices por sí mismas y otras necesitan estar rodeados de otras condiciones para alcanzar la felicidad. La forma de ser, la personalidad, la gente que les rodea y las vivencias que cada uno tenga en relación a su condición de peso, parecen ser los determinantes de la felicidad. En los siguientes párrafos se confirma que las emociones de la persona con obesidad no siempre son negativas, ya que eso llevaría a pensar que si adelgaza será feliz, como lo muestran las siguientes categorías encontradas.

\section{- Despreocupación y comodidad}

Los participantes declararon estar contentos y cómodos con el tamaño de sus cuerpos, de su salud, y de su estilo de vida, así como también afirmaron el tener relaciones sociales positivas. Frases tales como, "Eso no importa", "No me preocupo por mi peso", "No tengo planes relacionados con mi peso" o "Vivo mi vida feliz día a día", fueron escuchadas en los participantes del estudio confirmando que en general, estos no tienen como una prioridad entre sus planes principales el perder peso, pero si vivir la vida intensamente.

Mis metas son terminar el colegio y andar activo en la iglesia, pero el bajar de peso no es para mí una prioridad, como tengo tantas cosas que para mí lo del peso no le doy mucho sentimiento (Jonás, 128 kilos).

\section{- Me siento bien conmigo mismo}

Los componentes de la percepción y la actitud son dos dominios principales de la imagen del cuerpo (Thompson 1999). El componente de la percepción se refiere a la 
precisión con la que los individuos perciben su cuerpo, mientras que el componente de actitud se refiere a los pensamientos, sentimientos y reacciones de comportamiento sobre el propio cuerpo. Estos dos elementos fueron sin duda los que predominan en los discursos de los participantes. "Me siento bien, me veo bonita pero me gustaría bajar de peso".

Yo soy muy tranquila,.....me gusta pintar, no me gusta caminar ni nada de eso, me gusta mucho oír música, comer pasteles, y en relación a mi imagen corporal....pos me siento muy gordita pero muy bonita, no por lo gordita dejo de ser bonita, yo sé que estoy demasiado gorda pero me veo bonita, y si, me siento bien conmigo misma. (July, 135 kilos)

Al parecer ellos asocian el no poseer una enfermedad, y estar activos físicamente con sentirse bien con su peso, y eso es expresado por Carmen (138 kilos):

Mi sobrepeso no me molesta, me siento bien, yo siento que si no me siento mal físicamente, estoy bien; aunque si pienso, a veces pienso que estoy en sobrepeso pero que yo diga que me veo fea, no!!!, ¡Si, yo me veo y me siento bien!.

Una pregunta medular en este estudio fue: ¿Cómo te describirías a ti mismo (a)? En sus respuestas se encontraron una serie de características positivas muy relacionadas con la imagen del cuerpo y de la percepción que de sí mismos tienen todos ellos. Entre las características positivas, ellos afirmaron ser personas felices y buenas; trabajadoras, nobles, sinceras, líderes y siempre deseosas de ser ganadores o ser el "número uno". Los siguientes párrafos nos muestran lo anterior:

Yo soy "funny" (divertido): "outgoing" (sociable): buena persona, me llevo muy bien con otras personas, no soy muy callado pero tampoco soy muy hablador (Lucas, 185 kilos).

\section{- Divertido y feliz}

La identidad se construye en base a los discursos que las personas se dicen de sí mismas, pero que son producto de un discurso más amplio, social y predominante en una cultura determinada. Una característica personal que predominó en los discursos fue el ser extrovertidos; parece que el ser abierto y comunicativo, es una tendencia de aquellos con sobrepeso excesivo. Wally, una mujer Mexicoamericana, cuando se le preguntó sobre cómo se describiría a sí misma, alude a pensamientos, actividades sociales e incluso ideas y sentimientos de sí misma, como se aprecia en el siguiente párrafo:

Wally es amistosa, me gustan mucho las amistades, soy dedicada, me gusta que la gente tenga opiniones diferentes a las mías, me gusta la controversia, porque uno aprende de la diferencia de la gente, me gusta ver que es lo que piensa uno y otro, me gusta platicar con blancos, negros, puertorriqueños, indonesios... me gusta ser de respeto y juntarme en ambientes de muchas culturas. Soy muy demandante con mis cosas. Yo no me siento mal porque estoy gorda, mi ropa la escojo cuidadosamente cuando salgo de mi casa; de hecho la gente siempre me dice: iTe ves muy bien!, "iEres muy bonita! (Wally, 145k). 
Yo me describiría más bien divertido. Tengo muchos amigos y siempre me siento bien (Nico, 230 k).

Paquette y Raine (2004) por su parte, afirman que crear un medio ambiente de mayor apoyo desarrolla una imagen corporal positiva. La mayoría de los participantes en el estudio han aprendido a vivir con su condición de sobrepeso de manera positiva ayudados por un fuerte soporte de los miembros de su familia y por las características intrapersonales que ellos poseen.

Yo soy de los que siempre "me ha valido gorro", si yo fuera de los que me decepcionara mucho... o de los que me entrara la depresión y de que jhay nadie me quiere! a lo mejor hubiera sido un motivo para bajar de peso, pero como siempre "me ha valido gorro" yo creo que ha de ser por eso de que heee!!! ¡El que me quiera así como soy, si no ni modo, con mis amigos y con mi familia soy feliz! (Adelaido, 178 kilos)

En el estudio encontramos que aquellos que durante algún tiempo estuvieron o están actualmente casados, existe un conformismo hacia su imagen corporal, experimentando aprobación hacia su cuerpo, sobre todo al no verse limitados de alguna actividad física. Mizael ejemplifica esta afirmación:

Yo soy un tipo feliz, muy positivo, mi gordura no es de "nacencia" [desde que nació]... no es hereditaria, la he adquirido a través del tiempo y no te puedo decir que soy demasiado feliz gordo, pero no me causa ningún problema, hasta ahorita no hay ningún impedimento que trastorne mi vida emocional (Mizael, 240 kilos).

\section{- Gozo}

El ser humano goza con todo, y en este estudio los participantes afirman gozar de su vida, al igual que gozan la comida, y es que así lo aprendieron desde niños, a ellos les enseñaron a gozar la comida quizás sin límites, sin normas, sin leyes, pareciera ser que quiere todo para él, por eso se lo come, porque lo ama. Las palabras de Adelaido nos ilustran lo anterior:

Pues fue bonito, aquellas mesas grandes de tacos de harina en la mañana, una taza de café y 4 o 5 tortillas de harina recién paloteadas con mantequilla y vámonos a la escuela... fuimos 7 hermanos,... mi mamá paloteaba (hacía tortillas) todo el tiempo... calientitas... Eso y en combinación con el pan, siempre había pan de dulce, a eso de las 5 de la tarde: merienda obligatoria, tanto mi mamá como la familia de ella era capotear (agarrar) el pan.

Las respuestas favorables de estos participantes reflejan un cierto significado opuesto al que les provoca sufrimiento por subir de peso a causa de la comida. Las respuestas positivas plasmadas en este trabajo son las "verdades" de los participantes y en ese sentido, no las cuestionamos, sino tratamos de respetarlas y comprenderlas. No obstante, esa verdad es la que se muestra, es el contenido de la conciencia de los sujetos que hablan sobre el tema del sobrepeso y la obesidad. Es 
decir, no sabemos "lo que hay detrás", quizás ellos mismos tampoco lo sepan. Es posible que ellos se aferren a esas verdades para "mantenerse a flote" en una situación que es estigmatizante y a veces discriminativa como la obesidad. Finalmente, el interés de la investigación fue mostrar "el mundo de la vida" de nuestros participantes, un mundo que puede ser contradictorio, paradójico, como la realidad social misma.

\section{Reflexionesfinales. Una propuesta de intervención para el trabajo social}

Este trabajo presentó parte de los resultados de un estudio fenomenológico sobre la experiencia de ser una persona obesa donde se muestra cómo la imagen del cuerpo puede generar rechazo, estigma social, baja autoestima, inseguridad y emociones como la tristeza, frustración o vergüenza, y en contraparte, la aceptación de una persona con obesidad surge en una trama de relaciones familiares, donde ser obeso es menos importante que la calidad del vínculo, en donde la presencia de afecto y estimación por la persona con sobrepeso es más importante que las representaciones sociales dominantes de la obesidad.

Los discursos de los participantes mostraron cómo algunos trastornos alimenticios se gestan en la infancia, la cual en muchas ocasiones está llena de historias de tristeza, de sueños truncados y de anhelos insatisfechos o de mesas repletas de comida, donde el afecto y las emociones se trasmiten a través del alimento. El estudio demostró que una forma de desahogar la tensión y ante la inexperiencia para manejar las emociones se trasmiten a través del alimento, la persona recurre al consumo de alimentos como una forma de sentirse mejor ante situaciones estresantes. Evidentemente, al aumentar la cantidad de comida y de ciertos alimentos hipercalóricos, la dimensión del cuerpo aumenta, y con ello también las críticas, el aislamiento, y las emociones negativas.

En este trabajo cobra gran importancia la representación social que tiene para ellos la comida, asumiendo una fuerte carga emocional y mostrando la dificultad de renunciar a una de sus más importantes fuentes de gratificación o de escape en momentos de crisis. Alrededor de la comida, los entrevistados han escuchado muchas verdades, muchos sentidos comunes, desde la infancia, en la familia; después en la escuela o con los amigos y también los discursos institucionales, aquellos que ahora promueven disminuir el peso y cuidar la salud. En este sentido, los participantes, elaboran y entienden la obesidad de muchas y distintas maneras, a veces de forma contradictoria y paradójica.

Ante los hallazgos que el estudio arroja, se hace necesario que los trabajadores sociales rescatemos a través de la investigación, imágenes diferentes, historias y discursos alternativos que nos muestren una imagen del cuerpo más positiva, y si no es así, entonces darnos a la tarea de construir una imagen corporal en las personas con sobrepeso libre de cargas negativas que se promueva desde el interior de 
las propias familias, de los espacios educativos, de la sociedad en general, pero sobre todo desde ellas mismas.

En este sentido nuestra propuesta se basa en una intervención social con grupos de ayuda mutua, basada en la postura teórica del Interaccionismo Simbólico que enfatiza la centralidad desde el punto de vista del actor y la importancia de los significados comunes para entender e incidir en la acción social. De acuerdo a Pérez (2004), es importante entender desde este paradigma, que la realidad está constituida no solo por hechos observables y externos, sino también por significados, símbolos e interpretaciones elaboradas por el mismo sujeto a través de su interacción con los demás; en este sentido, la dinámica grupal, funge como un eficaz mecanismo de capacitación, que permite mejorar las habilidades necesarias para resolver los problemas, potenciando la capacidad de los participantes para interaccionar $y$ para ofrecer apoyo social en situaciones de incertidumbre. Además de favorecer el conocimiento de sí mismos y permitiendo la incorporación de nuevas habilidades que les permitan explorar y experimentar situaciones con la oportunidad de realizar un ajuste entre su autoimagen y la imagen que proyectan en los demás (Fernández y López 2006).

Los grupos de ayuda mutua como un modelo de intervención en trabajo social se sustentan en principios básicos como la solidaridad, igualdad, crecimiento, comprensión, fraternidad, honestidad y escucha, donde las actividades necesarias para el buen funcionamiento del grupo son tarea de todos. Esta intervención se enfoca principalmente a desarrollar las habilidades que se requieran para producir mejoras tanto en el bienestar personal como en el interpersonal.

La intervención propuesta no tendría el impacto esperado sin un tratamiento integral en el que se incluya aspectos psico-espirituales, socio-motivacionales, terapéuticos-emocionales y nutricios. Lo más importante será el cambio en las imágenes negativas, creencias no saludables y falsas representaciones sociales que las personas con obesidad mórbida tienen de sí mismas, de la comida, de la salud, y de su propia responsabilidad para cambiar la trama de sus vidas, hacia un un mayor desarrollo social integral.

A partir de los resultados de este estudio se propone utilizar técnicas alternativas donde liberen creencias negativas y emociones tóxicas, reaprehendiendo creencias saludables. La idea es lograr una intervención con un enfoque práctico y potente para lograr cambios personales tangibles. La opción del trabajo con grupos favorece el conocimiento de sí mismos y la incorporación de nuevas habilidades que les permitan explorar y experimentar situaciones con la oportunidad de realizar un ajuste entre su autoimagen y la imagen que proyectan en los demás (Fernández y López 2006).

El énfasis en este grupo estará puesto en motivar a los participantes para que aprovechen todas las oportunidades de ayuda mutua que se generen en el grupo. La 
función del trabajador social en este modelo desempeñaría un papel activo, que guíe y oriente en la adquisición de competencias y habilidades en los integrantes del grupo para que puedan conocerse e interiorizar determinadas creencias, desechar pensamientos y hábitos disfuncionales que imposibiliten y frenen su desarrollo personal e incorporar patrones de alimentación y activación física adecuados. En las interacciones dinámicas todos aportan ideas y opiniones. De ellas emergen nuevos patrones de pensamiento y conducta que producirán cambios en el comportamiento de cada persona en su proceso de adopción hacia un estilo de vida saludable.

\section{Referencias bibliográficas}

Álvarez-Gayou, J. 2003. Como hacer investigación cualitativa. México: Paidós.

Ball, K. and C. Lee. 2000. "Relationships between psychological stress, coping and disordered eating: A review". Psychology and Health 14, 1007-1035.

Bertrán, M. 2009. "Reflexiones sobre el análisis antropológico de la alimentación". En Salud, nutrición y alimentación: Investigación cualitativa, coordinado por E. Castro. Monterrey, México: Universidad Autónoma de Nuevo León.

Bilbao, I., J. March., Prieto, M. 2002. "Diez aportaciones del empleo de la metodología cualitativa en una auditoria de comunicación interna en atención primaria". Revista española de Salud Pública, 76 (5) 483-492. Consulta 19 de mayo de 2008 http://redalyc.uaemex.mx/redalyc/pdf/170/17076510.pdf

Cabello, L. 2010. Voces y vivencias de aquéllos que gozan y sufren la obesidad un estudio fenomenológico. Monterrey, México: Universidad Autónoma de Nuevo León.

Cabello, L., Zúñiga, J. 2007. "Aspectos intrapersonales y familiares asociados a la obesidad: un análisis fenomenológico". Ciencia. UANL 183-188.

Cabello, L., Ávila, N., Gara, G. 2011. "Obesidad: Los problemas de los grupos vulnerables". En La problemática de los grupos vulnerables: visiones de la realidad, coordinado por J. Acebedo y M. A. Pérez. Coahuila: Universidad Autónoma de Coahuila.

Caldwell, W., Kimball. C. 2001. Obesity sourcebook. United States: Omnigraphics.

Calleja, E. 2010. "Obesidad: Un enfoque multidisciplinario". En Ciencia al Día, coordinado por J.A. Morales (Coord). Universidad Nacional del Estado de Hidalgo.

Decaluwé, V., Braet, C., Fairburn. G. 2003. "Binge eating in obese children and adolescents". International Journal of Eating Disorders 33 (1): 10-12. Consulta 9 de Junio de 2003 (www.interscience.wiley.com)

Denzin, K., Lincoln. Y. 2003. "Introduction: The discipline and practice of qualitative research". In Collecting and interpreting qualitative materials, edited by K. Denzin and Y. Lincon. USA: Sage

Fabian, J., Thompson, K. 1989. "Body image and eating disturbance in young females". International Journal of Eating Disorders 8, 63-74.

Fernández, T., López. A. 2006. Trabajo social con grupos. Madrid: Alianza Editorial.

Fivush, R., Haden, C. (eds). 1997. Narrative and representing experience: Preschooler's developing autobiographical recounts. Mahwah, Nueva Jersey: Erlbaum.

Focault, M. 1979. "Verdad y poder." En Microfísica del poder. Madrid: La Piqueta. 
Gavilán, V. 2005. "Representaciones del cuerpo e identidad de género y ética en la población indígena del norte de Chile." Estudios Atacameños 30: 135-148. Consulta 15 de mayo de 2013 (http://www.scielo.cl/scielo.php?script=sci_arttext\&pid=S071810432005000200008)

Gergen, K. 2006. Construir la realidad. Barcelona: Paidos.

González, T. 2003. "El interaccionismo simbólico". Pp. 167-215 en Teoría sociológica moderna editado por S. Giner. Barcelona: Ariel.

González, F. 2007. "Investigación cualitativa y subjetividad". Pp.748-769 en Getting a life: The development of the life story in adolescence, editado por Habermas, T. and S. Bluck. México: McGraw Hill

Hymes, Dell. 1996. Ethnography, linguistics, narrative inequality: Toward an understanding of voice. Londres: Taylor and Francis.

Hussserl, E. 1965. Ideas relativas a una fenomenología pura y a una filosofía fenomenológica. México: Fondo de Cultura Económica.

Husserl, E. 1998. Invitación a la fenomenología. México: Paidós

Instituto Mexicano del Seguro Social. 2010. Encuesta Nacional de Coberturas. Consulta 16 de Enero de 2010 (http://www.imss.gob.mx/publicaciones/salud/enco.htm).

Lazarus, R. 2000. Estrés y emoción. Manejo e implicaciones en nuestra salud. Bilbao: Descleé de Brouwer.

Lunner, K., H. Werthem., K. Thompson., J. Paxton., F. McDonald. and S. Halvaarson. 2000. "A cross-cultural examination of weight-related teasing, body image, and eating disturbance in Swedish and Australian samples". Pp. 430-435 en International Journal of Eating Disorders, editado por Wiley, J. and I. Sons. USA: New York.

Martínez, A. 2004. "La construcción social del cuerpo en las sociedades contemporáneas". Papers 73, 127-152.

Maxwell, A. 2009. "Understanding and Validity in Qualitative Research". Harvard Educational Review 62 (3): 279-300.

Mruk, C. J. 1999 . Self-esteem: Research, theory, and practice. 2nd ed. New York: Springer.

McFarland, B., Backer-Bauman, T. 1988. Feeding the empty heart: Adult children and compulsive eating. Cambridge, MA: A Harper/Hazelden Book.

Moreno, D. 2000. "La Investigación Cualitativa en Salud". Revista Salud Pública y Nutrición, 1 (2). Consulta 19 de mayo de 2008 de (http://www.uanl.mx/publicaciones/respyn/i/2/ ensayos/investigacion cualitativa.html).

Olaiz, G., J. Rivera., T. Shamah., R. Rojas., S. Villalpando., M. Hernandez., and J. Sepulveda. 2007. Encuesta Nacional de Salud y Nutrición 2006. Cuernavaca, Morelos: Instituto Nacional de Salud Pública.

Paquette, C., Raine, K. 2004. "Sociocultural context of women's body image". Social Science and Medicine, 59 (5), 1047-1058.

Pelican, S., F. Vanden, B. Holmes., L. Melcher., M. Wardlaw., M. Raidl., B. Wheler., and S. More. 2005. "The Power of Others to Sahpe our Identity: Body Image, Physical Abilities, and Body Weight". Family and consumer Sciences Research Journal 34. Consulta 3 de febrero de 2010 (http://fcs.sagepub.com).

Pérez, G. 2004. Investigación cualitativa: retos e interrogantes. I. Métodos. España: La Muralla. 
Pérez, S., Vega-García, A., Romero-Juárez, G. 2007. "Prácticas alimentarias de mujeres rurales: ¿una nueva percepción del cuerpo?". Salud pública de México 49 (1), 52-62.

Polkinghorne, D. 2004. Practice and the human sciences: The case for a judgmentbased practice of care. Albany: State University of New York Press.

Pollio, R., Henley, T. Thompson, B. 1997. The Phenomenology of Everyday Life. Cambridge: Cambridge University Press.

Ramirez, B., Anzaldúa, R. 2005. Subjetividad y relación educativa. México: Universidad Autónoma Metropolitana Azcapotzalco.

Rodríguez, G., Gil, J., García, G. 1999. Metodología de la investigación. Granada: Aljibe.

Sedó, P. 2005. "Significados y prácticas de alimentación de un grupo de personas adultas mayores diabéticas y sus familiares, en el cantón de La Unión, Cartago". Anales en Gerontología (5), 39-53.

Shamah-Levy, T., Villalpando-Hernández, S., Rivera-Dommarco, J. 2007. Resultados de Nutrición de la ENSANUT 2006. Cuernavaca, México: Instituto Nacional de Salud Pública.

Thompson, K., Heinberg, J. 1999. "The media's influence on body image, disturbance and eating disorders: We've reviled them, now can we rehabilitate them". Journal of Social Issues 55 (2), 339-353.

Ungar, M. 2003. "Qualitative contributions to resilience research". Qualitative Social Work, 2 (1), 85-102.

Vázquez, S., Cabello, M., and Montemayor, S. 2010. "La obesidad infantil: más que una cuestión de alimentación". En Obesidad y prácticas alimentarias: impactos a la salud desde una visión multidisciplinaria, editado por Cabello, M y S. Garay. México: Universidad Autónoma de Nuevo León.

Von Eckartsberg, R. 1998. "Introducing existential-phenomenological psychology". Pp 320 In Phenomenological Inquiry in Psychology, editing by Valle, R. New York: Plenum.

Organización Mundial de la salud. 2010. Cifras y datos. 10 datos sobre la obesidad. Consulta el 20 de abril de 2011. (http://www.who.int.features/factfiles/obesity/es).

World Health Organization. 2010. "Sitio oficial de WHO en español." Consulta 23 de marzo de 2012 (http://www.who.int/topics/obesity/es/). 


\section{ARTICULOS/ARTICLES}

Empresas spin-off y género: diferencias entre hombres y mujeres en la creación de empresas de base tecnológica / Spin-off and gender: differences between men and women in the creation of technology-based companies

Ignasi Brunet Icart y Joan Rodríguez Soler

Custodia compartida: atribución vivienda familiar / Shared custody: the acquisition the family living Beatriz Escudero Berzal

Aproximación al estado de bienestar en Polonia / Aproximation to the welfare state in Poland Paloma Serrano Postigo

Menores víctimas de la violencia de género: propuesta de proyecto educativo / Minours who are victims of gender-violence. Proposal for an educational project

Luis Manuel Rodríguez Otero

La obesidad paradójica: construcción de una imagen corporal contradictoria / Paradoxical obesity: building a contradictory body image

Martha Leticia Cabello Garza y María Concepción Arroyo Rueda

\section{RESENAAS/REVIEWS}

Del Fresno García, M., Segado Sánchez-Cabezudo, S., López Peláez, A. (eds). Trabajo social con comunidades en el siglo XXI / Social Work with communites in the XXI Century (por Yolanda Meneses García)

Sagrario Segado Sánchez-Cabezudo, Miguel del Fresno García y Antonio López Peláez (eds.). Modelos de Trabajo Social con grupos: nuevas perspectivas y nuevos contextos / Models of social work with groups: new perspectives and new contexs (por Emilio Díaz de Mera)

Antonio López Peláez (ed.) The Robotics Divide: a New Frontier in the 21st Century? / La brecha robótica: ¿una nueva frontera en el siglo XXI? (por Raquel Pérez García)..... 